\title{
Método Extendido de Estados Correspondientes para la Predicción de Densidades de Gas Natural Licuado
}

\section{Pedro A. Alonso-Dávila, Sonia H. Soriano-Pérez y Francisco Sánchez-Mares}

Universidad Autónoma de San Luis Potosí, Facultad de Ciencias Químicas, Centro de Investigación y Estudios de Posgrado, Av. Dr. Manuel Nava \# 6, 78210 San Luis Potosí, SLP-México

(e-mail: palonsod@uaslp.mx, sorianos@uaslp.mx)

\section{Resumen}

En este trabajo se presenta un método basado en el principio de estados correspondientes para predecir la densidad de un fluido con un mínimo de información. El método se basa en una formulación molecular del principio de estados correspondientes, utilizando metano como fluido de referencia. Las densidades de las distintas mezclas de gas natural se calcularon a temperaturas entre 95 y $150 \mathrm{~K}$ y se compararon con datos experimentales y estimaciones de densidad de ocho ecuaciones de estado y dos correlaciones. Este método de estados correspondientes es la correlación más exacta y confiable para predecir densidades de líquido saturado, presenta desviaciones menores al $0.1 \%$ para gas natural y menores al $0.2 \%$ para gas licuado de petróleo.

Palabras clave: estados correspondientes, gas natural, ecuación de estado, densidad

\section{Extended Corresponding States Model for Predictions Liquefied Natural Gas Densities}

\begin{abstract}
This paper presents a method based on the corresponding states principle for predicting the density of a fluid given a minimum amount of information. The method is based on a molecular formulation of the principle of corresponding states, using methane as a reference fluid. The densities of the various natural gas mixtures were calculated at temperatures between 95 and $50 \mathrm{~K}$ and compared with experimental data and with predictions of two correlations and eight equations of state. The proposed corresponding states method is the most accurate and reliable correlation to predict saturated liquid densities, and gives deviations lower than $0.1 \%$ for natural gas and less than $0.2 \%$ for liquefied petroleum gas.
\end{abstract}

Keywords: corresponding states, natural gas, equation of state, density 


\section{INTRODUCCION}

El gas natural es una mezcla de hidrocarburos ligeros que contiene principalmente metano, pequeñas cantidades de etano, propano, y otros hidrocarburos hasta $\mathrm{C}_{6}$ y en proporciones variables nitrógeno, dióxido de carbono y ácido sulfhídrico. El gas licuado de petróleo es una mezcla que contiene principalmente propano y butano y pequeñas cantidades de otros componentes. El gas natural es un combustible limpio que se puede emplear en sectores tan diversos como la industria química, la generación de energía eléctrica, etc. El gas natural se transporta en grandes volúmenes principalmente a través de gaseoductos, buques y tanques criogénicos de alta presión.

La predicción confiable de las propiedades termodinámicas del gas natural es muy importante para la extracción, transporte y procesamiento. La densidad del líquido saturado en algunas aplicaciones debe calcularse con una desviación menor al 0.1\% (McCarty, 1982), por ejemplo, el flujo másico de gas natural en gasoductos requiere de datos precisos de densidad.

Dada la importancia de la densidad de Gas Natural Licuado se han propuesto varias correlaciones. McCarty (1982), estudio y reportó los resultados de predicciones de densidad de tres correlaciones: un modelo extendido de estados correspondientes (Rowlinson y Watson 1969), un modelo de esferas duras de Rodosevich y Miller (1974) y el método empírico de Klosek y McKinley (1968) que calcula la densidad como función de temperatura y composición. Los tres métodos predicen la densidad de gas natural licuado con una exactitud dentro de $0.1 \%$.

Se han hecho muchos esfuerzos para desarrollar correlaciones y aplicar ecuaciones de estado cúbicas a la predicción densidades de líquido saturado a sistemas de gas natural. Spencer y Danner (1972), modificaron la ecuación de Rackett para calcular la densidad de líquido saturado de componentes puros y mezclas, esta ecuación falla a temperaturas de la mezcla próximas a la temperatura crítica de uno de los componentes. Iglesias-Silva y Hall (1997), desarrollaron una correlación para calcular la densidad de líquido saturado de refrigerantes halogenados puros, Nasrifar et al. (1999) extendieron esta correlación a mezclas y calcularon densidades de gas natural con gran precisión. Posteriormente, Eslami (2001) desarrolló una ecuación de estado analítica con base molecular para predecir densidades de gas natural con una desviación absoluta mayor del $2 \%$. Una descripción detallada de las correlaciones de Spencer y Danner e Iglesias-Silva y Hall se puede consultar en el trabajo de Nasrifar et al. (1999; 2000).

Nasrifar y Bolland (2006), usaron 10 ecuaciones de estado para predecir propiedades termodinámicas de mezclas de gas natural. Entre las ecuaciones de estado cúbicas que probaron se encuentran Redlich-Kwong-Soave (RKS) (Soave, 1972), Peng-Robinson (PR) (Peng y Robinson, 1976) y la ecuación de estado de Nasrifar-Moshfegian (NM) (Nasrifar y Moshfegian, 2002). Para las predicciones de densidades de líquido saturado, ellos reportaron la desviación absoluta promedio (AAD) global: $1.74 \%$ para RKS, $10.76 \%$ para PR y $1.16 \%$ para NM.

Recientemente Janish et al. (2007), hicieron cálculos del equilibrio de fases y densidad de líquido saturado, con las ecuaciones de estado por contribución de grupos, PSRK (SRK predictivo) y VTPR (PR de volumen trasladado) que combinan ecuaciones de estado cúbicas con UNIFAC para obtener un modelo predictivo confiable. Las predicciones de densidad de líquido saturado se compararon con datos experimentales de tres sistemas binarios: $\mathrm{N}_{2}+\mathrm{CH}_{4}, \mathrm{CH}_{4}+\mathrm{C}_{2} \mathrm{H}_{6}$ y $\mathrm{N}_{2}+\mathrm{C}_{2} \mathrm{H}_{6}$ y se encontró que la desviación absoluta promedio global fue: 4.4\% para PSRK, 2.1\% para VTPR y $8.1 \%$ para PR.

La teoría de estados correspondientes permite predecir las propiedades de un fluido con un mínimo de información: típicamente se requieren las propiedades del fluido de referencia, parámetros de reducción del fluido problema y parámetros específicos de estados correspondientes. El método está basado en una formulación molecular del principio de estados correspondientes (Mollerup y Rowlinson, 1974) usando metano como fluido de referencia y se implementaron los recientes desarrollos de Estela-Uribe y Trusler (2001) para representar los factores de forma molecular de los hidrocarburos relativos a esta referencia. La predicción confiable de densidades de gas natural es de gran importancia para la industria, pero, pocos métodos la calculan de una manera eficiente. 


\section{ESTADOS CORRESPONDIENTES}

Dos sustancias puras están en estados correspondientes si se pueden modelar por un par de ecuaciones que relacionan dos funciones adimensionales de las propiedades configuracionales (Rowlinson y Watson, 1969, Mollerup y Rowlinson, 1974, y Estela-Uribe y Trusler, 2001). Si la presión y temperatura se seleccionan como variables independientes:

$Z_{\alpha}[P, T]=Z_{o}\left[\frac{P h_{\alpha \alpha, o}}{f_{\alpha \alpha, o}}, \frac{T}{f_{\alpha \alpha, o}}\right] \quad G_{\alpha}[P, T]=f_{\alpha \alpha, o} G_{o}\left[\frac{P h_{\alpha \alpha, o}}{f_{\alpha \alpha, o}}, \frac{T}{f_{\alpha \alpha, o}}\right]-R T \ln \left(h_{\alpha \alpha, o}\right)$

$G_{\alpha}$ es la energía libre de Gibbs configuracional del componente puro $\alpha$ a $P$ y $T$ y $G$ o es la energía libre de Gibbs de la sustancia de referencia $o$, que es similar a $\alpha$ a presión y temperatura reducidas $P h_{\alpha \alpha, o} / f_{\alpha \alpha, o,} T / f_{\alpha \alpha, o} . Z_{\alpha}$ es el factor de compresibilidad de $\alpha$ y $Z_{o}$ lo es del componente puro $o$.

Para que el principio de estados correspondientes tenga aplicación práctica, se requiere una correlación para los factores de escala $f_{\alpha \alpha, o}$ y $h_{\alpha \alpha, o}$, que permita extenderlo a sustancias puras de propiedades desconocidas. Esto se ha hecho para sustancias no polares y no asociantes que tienen ecuaciones de estado reducidas similares. Los factores de de escala $f_{\alpha \alpha, o}$ y $h_{\alpha \alpha, o}$ son funciones débiles de temperatura y volumen y sus valores son cercanos al cociente de las constantes críticas. Las desviaciones entre esos valores se han incorporado en los factores de forma $\theta$ y $\Phi$, desarrollados por Leach et al. (1968):

$$
f_{\alpha \alpha, o}=\left(T_{\alpha \alpha}^{c} / T_{o}^{c}\right) \theta_{\alpha \alpha, o}\left[V_{r}, T_{r}\right] \quad h_{\alpha \alpha, o}=\left(V_{\alpha \alpha}^{c} / V_{o}^{c}\right) \Phi_{\alpha \alpha, o}\left[V_{r}, T_{r}\right]
$$

donde $V_{r}=V / V_{\alpha \alpha,}^{c}, \quad T_{r}=T / T^{c}$

Los factores de forma son parámetros dependientes de la temperatura que multiplican a las propiedades críticas de un fluido para obtener así las variables reducidas adecuadas. Estela-Uribe y Trusler $(1998,2001)$ desarrollaron una nueva versión de este método y determinaron los factores de forma por regresión de datos experimentales. Otras propiedades termodinámicas se calculan de las ecuaciones (1) y (2) por medio de relaciones bien conocidas de la termodinámica clásica. La precisión de cualquier modelo de estados correspondientes depende de la correcta aplicación de la correspondencia entre los componentes de la mezcla y el fluido de referencia, la aplicación de un modelo adecuado de mezclado y el uso de una ecuación de estado para el fluido de referencia muy exacta. La selección de la ecuación de estado de referencia es fundamental para lograr la mayor exactitud del método, por esta razón se utilizó la ecuación de estado de Setzmann y Wagner (1991).

Para aplicar el principio de estados correspondientes a mezclas se adopta el modelo de van del Waals de un fluido tal como fue propuesto por Leland et al. (1968, 1969). En esta aproximación la energía libre de Gibbs de la mezcla después de restar el término de mezclado ideal es igual a la de una sustancia "pura" equivalente. Esta sustancia es diferente para cada mezcla y se indicará con el subíndice $x$. Sus propiedades se calculan a partir de las propiedades de la sustancia de referencia $o$.

La energía libre de Gibbs configuracional de la mezcla $G_{m}$ es entonces:

$$
\begin{aligned}
& G_{m}[P, T, x]=G_{x}[P, T, x]+R T \sum_{\alpha} x_{\alpha} \ln x_{\alpha} \quad G_{x}[P, T, x]=f_{x} G_{o}\left[P h_{x} / f_{x}, T / f_{x}\right]-R T \ln h_{x} \\
& \text { у } \quad Z_{m}[P, T, x]=Z_{x}[P, T, x]=Z_{o}\left[P h_{x} / f_{x}, T / f_{x}\right]
\end{aligned}
$$

De acuerdo a la aproximación de van der Waals de un fluido, los parámetros de reducción son:

$$
h_{x}=\sum \sum x_{\alpha} x_{\beta} h_{\alpha \beta, o} \quad f_{x} h_{x}=\sum \sum x_{\alpha} x_{\beta} f_{\alpha \beta, o} h_{\alpha \beta, o}
$$

donde $\alpha$ y $\beta$ son componentes de la mezcla $(\alpha \neq \beta)$. Los parámetros de interacción binaria son 


$$
\begin{array}{ll}
f_{\alpha \beta, o}=\xi_{\alpha \beta}\left(f_{\alpha \alpha, o} . f_{\beta \beta, o}\right)^{1 / 2} & h_{\alpha \beta, o}=\eta_{\alpha \beta}\left(\frac{1}{2} h_{\alpha \alpha, o}^{1 / 3}+\frac{1}{2} h_{\beta \beta, o}^{1 / 3}\right) \\
f_{\alpha \beta, o}=\frac{T_{\alpha \beta}^{c}}{T_{o}^{c}} \theta_{\alpha \beta, o}\left[\frac{V \Phi_{\alpha \beta, o}}{V^{c} o^{h x}}, \frac{T \theta_{\alpha \beta, o}}{T^{c} o^{f x}}\right] & h_{\alpha \beta, o}=\frac{V_{\alpha \beta}^{c}}{V_{o}^{c}} \Phi_{\alpha \beta, o}\left[\frac{V \Phi_{\alpha \beta, o}}{V^{c} o^{h x}}, \frac{T \theta_{\alpha \beta, o}}{T^{c} o^{f x}}\right]
\end{array}
$$

Las ecuaciones (6) y (7) representan, a nivel microscópico, la interacción entre moléculas diferentes en la mezcla. Los parámetros de interacción binaria $\xi$ y $\eta$ tienen valores cercanos a la unidad y se determinan de datos experimentales de sistemas binarios, en esta extensión se consideran independientes de volumen, temperatura y composición. Mollerup y Rowlinson (1974) y Mollerup (1977) utilizaron la ecuación de estado de Goodwin para modelar la sustancia de referencia (metano) y los factores de forma de Leach et al. (1968). En este trabajo se utilizó la ecuación de estado de Setzmann y Wagner (1991), los factores de forma de Estela-Uribe y Trusler (2001) y los valores de $\xi$ y $\eta$ se ajustaron a datos experimentales para los componentes que se encuentran en el gas natural y gas del petróleo,

\section{RESULTADOS Y DISCUSION}

El programa de cálculo del método extendido de estados correspondientes fue escribió en FORTRAN y se implemento en sistemas Linux y Windows. Este método fue utilizado por Mollerup (1977), predice la entalpía, entropía y propiedades $P-V$ - $T$ saturadas o fuera de la línea de saturación; y también predice el equilibrio líquido vapor de sustancias puras y mezclas que pueden contener los siguientes componentes: metano, etano, etileno, propano, propileno, butano, isobutano, pentano, isopentano, nitrógeno, monóxido de carbono, dióxido de carbono y sulfuro de hidrógeno.

Para el cálculo de densidades de mezclas de gas natural licuado y gas licuado de petróleo, se usaron los datos experimentales de densidad de líquido saturado, reportados por McCarty (1982) en el rango de temperatura de $105-140 \mathrm{~K}$, los resultados se presentan en la Tabla 1 . El método extendido de estados correspondientes predice la densidad de gas natural licuando y gas licuado de petróleo con desviaciones menores a $0.1 \%$ y $0.2 \%$ respectivamente. La Figura 1 presenta la desviación porcentual promedio (\%Bias) de las predicciones de densidad por estados correspondientes como una función de la presión, se observa que la mayor parte de los datos experimentales satisfacen el requerimiento de desviaciones menores al $0.2 \%$ para LPG y al $0.1 \%$ para GNL. La mayor desviación para la mezcla 5 se debe a que la incertidumbre experimental de esa mezcla es mayor, según indica McCarty (1982). En las mezclas 8 y 12 de propano-nitrógeno y metano-nbutano-nitrógeno, la desviación se incrementa con el contenido de nitrógeno y mayor presión.

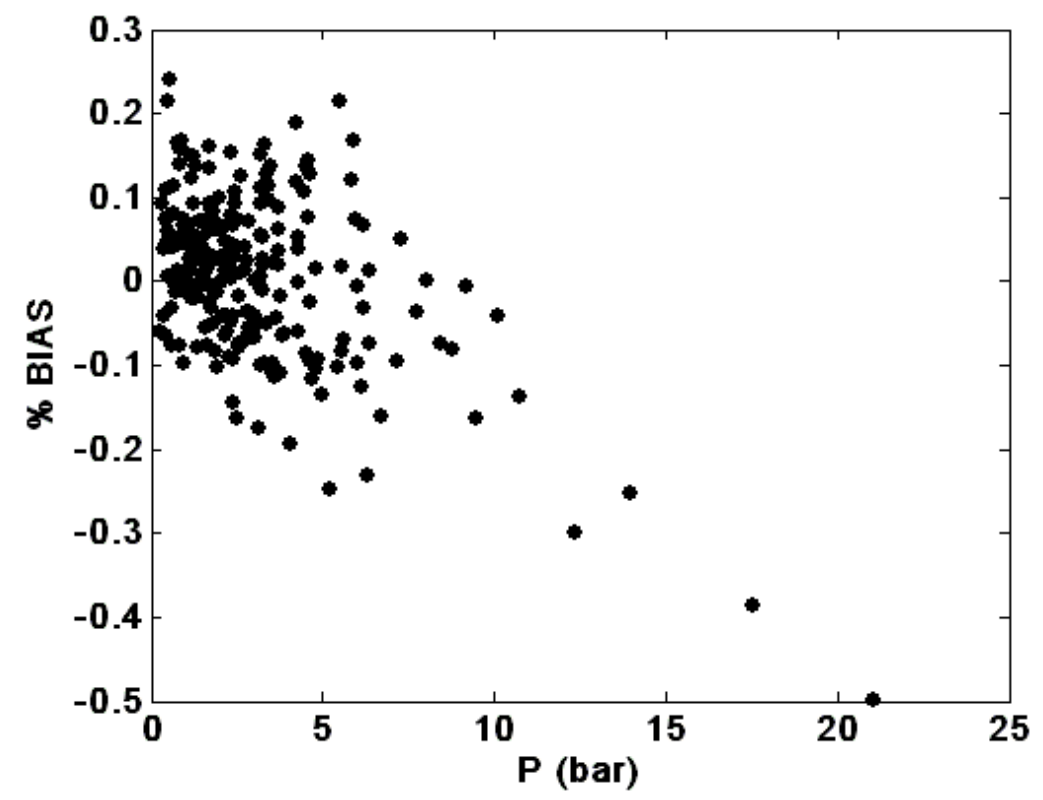

Fig. 1: Desviación de densidad de líquido saturado para todas los sistemas de la Tabla1 
Tabla 1: Resultados de cálculos de densidad de mezclas de GNL Y GLP por el método extendido de estados correspondientes (MEEC), las correlaciones de Iglesias-Silva y Hall (ISH), Rackett y Spencer-Danner (RSD) y la ecuación de estado de Eslami.

\begin{tabular}{|c|c|c|c|c|c|c|c|c|c|c|}
\hline & Sistema & & rango & MEEC & & ISH & & RSD & & Eslami \\
\hline & GNL & np & $\mathrm{T}(\mathrm{K})$ & \%BIAS & $\% A A D$ & \%BIAS & $\% A A D$ & \%BIAS & \%AAD & \%AAD \\
\hline 1 & $\mathrm{C}_{1}+\mathrm{C}_{2}$ & 21 & $105-140$ & 0.01 & 0.04 & -0.32 & 0.32 & -0.28 & 0.28 & 2.04 \\
\hline 3 & $\mathrm{C}_{1}+\mathrm{C}_{3}$ & 20 & $105-130$ & 0.00 & 0.05 & 0.06 & 0.16 & -0.26 & 0.50 & 3.64 \\
\hline 4 & $\mathrm{C}_{1}+\mathrm{nC}_{4}$ & 31 & $105-140$ & 0.02 & 0.08 & -0.27 & 0.45 & -0.41 & 0.99 & 2.17 \\
\hline 5 & $\mathrm{C}_{1}+\mathrm{iC}_{4}$ & 17 & $110-140$ & 0.08 & 0.12 & 0.79 & 0.89 & 0.38 & 0.92 & 2.24 \\
\hline 6 & $\mathrm{C}_{1}+\mathrm{N}_{2}$ & 21 & $95-140$ & -0.01 & 0.13 & 1.21 & 1.21 & 1.00 & 1.04 & 1.57 \\
\hline 7 & $\mathrm{C}_{2}+\mathrm{N}_{2}$ & 4 & $105-120$ & -0.07 & 0.07 & -0.15 & 0.15 & -0.33 & 0.33 & 0.55 \\
\hline 8 & $\mathrm{C}_{3}+\mathrm{N}_{2}$ & 6 & $100-115$ & -0.14 & 0.14 & -0.93 & 0.93 & -1.13 & 1.13 & 1.42 \\
\hline 9 & $\mathrm{C}_{1}+\mathrm{C}_{2}+\mathrm{C}_{3}$ & 10 & $105-125$ & 0.09 & 0.09 & -0.01 & 0.09 & -0.39 & 0.55 & \\
\hline 10 & $\mathrm{C}_{1}+\mathrm{C}_{2}+\mathrm{N}_{2}$ & 7 & $105-120$ & -0.08 & 0.10 & 0.81 & 0.81 & 0.99 & 0.99 & \\
\hline 11 & $\mathrm{C}_{1}+\mathrm{C}_{3}+\mathrm{iC}_{4}$ & 3 & $110-115$ & 0.05 & 0.05 & & & & & \\
\hline 12 & $\mathrm{C}_{1}+\mathrm{nC}_{4}+\mathrm{N}_{2}$ & 4 & $110-125$ & -0.19 & 0.19 & 0.72 & 0.72 & 1.40 & 1.40 & 2.89 \\
\hline 13 & $\mathrm{C}_{1}+\mathrm{C}_{2}+\mathrm{C}_{3}+\mathrm{nC}_{4}$ & 11 & $110-135$ & 0.03 & 0.03 & -0.11 & 0.20 & 0.62 & 0.62 & 3.39 \\
\hline 14 & $\mathrm{C}_{1}+\mathrm{C}_{2}+\mathrm{C}_{3}+\mathrm{iC}_{4}$ & 11 & $110-135$ & 0.05 & 0.05 & -0.06 & 0.18 & 0.80 & 0.80 & 3.26 \\
\hline 15 & $\mathrm{C}_{1}+\mathrm{C}_{2}+\mathrm{C}_{3}+\mathrm{N}_{2}$ & 4 & $105-120$ & -0.05 & 0.05 & 0.81 & 0.81 & 0.99 & 0.99 & \\
\hline 16 & $\mathrm{C}_{1}+\mathrm{C}_{2}+\mathrm{C}_{3}+\mathrm{nC}_{4}+\mathrm{iC} \mathrm{C}_{4}$ & 11 & $105-130$ & 0.07 & 0.08 & -0.06 & 0.23 & 0.38 & 0.39 & 2.89 \\
\hline 17 & $\mathrm{C}_{1}+\mathrm{C}_{2}+\mathrm{C}_{3}+\mathrm{nC}_{4}+\mathrm{N}_{2}$ & 7 & $105-130$ & -0.08 & 0.09 & 0.40 & 0.43 & 0.74 & 0.74 & 3.26 \\
\hline 18 & $\mathrm{C}_{1}+\mathrm{C}_{2}+\mathrm{C}_{3}+\mathrm{iC}_{4}+\mathrm{N}_{2}$ & 9 & $105-130$ & -0.05 & 0.06 & 0.65 & 0.65 & 0.82 & 0.82 & 3.28 \\
\hline 19 & $\begin{array}{l}\mathrm{C}_{1}+\mathrm{C}_{2}+\mathrm{C}_{3}+\mathrm{nC}_{4}+\mathrm{iC}_{4}+ \\
\mathrm{N} 2\end{array}$ & 15 & $105-130$ & 0.04 & 0.05 & -0.01 & 0.33 & 0.48 & 0.48 & 2.43 \\
\hline 20 & $\begin{array}{l}\mathrm{C}_{1}+\mathrm{C}_{2}+\mathrm{C}_{3}+\mathrm{nC}_{4}+\mathrm{iC}_{4}+ \\
\mathrm{nC}_{5}+\mathrm{iC}_{5}\end{array}$ & 9 & $110-130$ & 0.01 & 0.01 & 0.31 & 0.42 & 0.62 & 0.64 & 3.26 \\
\hline \multirow[t]{3}{*}{21} & $\begin{array}{l}\mathrm{C}_{1}+\mathrm{C}_{2}+\mathrm{C}_{3}+\mathrm{nC}_{4}+\mathrm{iC}_{4}+ \\
\mathrm{nC}_{5}+\mathrm{iC}_{5}+\mathrm{N}_{2}\end{array}$ & 13 & $110-130$ & 0.03 & 0.04 & -0.05 & 0.18 & 0.27 & 0.28 & 3.28 \\
\hline & \multirow{2}{*}{$\begin{array}{l}\text { Promedio } \\
\text { GLP }\end{array}$} & & & 0.01 & 0.07 & 0.19 & 0.46 & 0.33 & 0.69 & 2.42 \\
\hline & & np & $\mathrm{T}(\mathrm{K})$ & \%BIAS & \%AAD & \%BIAS & \%AAD & \%BIAS & \%AAD & \%AAD \\
\hline 22 & $\mathrm{C}_{2}+\mathrm{C}_{3}$ & 12 & $105-140$ & 0.13 & 0.13 & -0.59 & 0.59 & -0.77 & 0.77 & 1.71 \\
\hline 23 & $\mathrm{C}_{2}+\mathrm{nC}_{4}$ & 8 & $110-140$ & -0.09 & 0.09 & 0.47 & 0.47 & -0.85 & 0.85 & 2.94 \\
\hline 24 & $\mathrm{C}_{2}+\mathrm{iC}_{4}$ & 6 & $105-130$ & -0.04 & 0.05 & 0.16 & 0.16 & -0.84 & 0.84 & 3.19 \\
\hline 25 & $\mathrm{C}_{3}+\mathrm{nC}_{4}$ & 8 & $110-150$ & 0.01 & 0.02 & -1.08 & 1.08 & -0.78 & 0.78 & 3.75 \\
\hline 26 & $\mathrm{C}_{3}+\mathrm{iC}_{4}$ & 8 & $105-130$ & -0.07 & 0.07 & -1.32 & 1.32 & -0.95 & 0.95 & 3.07 \\
\hline 27 & $\mathrm{nC}_{4}+\mathrm{iC}_{4}$ & 4 & $125-140$ & -0.19 & 0.19 & -1.45 & 1.45 & -0.64 & 0.64 & 3.19 \\
\hline & Promedio & & & -0.06 & 0.14 & -0.59 & 0.80 & -0.81 & 0.81 & 2.84 \\
\hline
\end{tabular}

Los cálculos de las desviaciones de densidad de realizaron utilizando las ecuaciones siguientes:

$$
\mathrm{AAD} \%=\sum\left|\frac{\rho_{\text {exp }}-\rho_{\text {calc }}}{\rho_{\text {exp }}}\right| \cdot \frac{100}{N} \quad \mathrm{BIAS \%}=\sum \frac{\rho_{\text {exp }}-\rho_{\text {calc }}}{\rho_{\text {exp }}} \cdot \frac{100}{N}
$$

El método de estados correspondientes produce el mejor ajuste con los datos experimentales de densidades de líquido saturado, menor a $0.1 \%$ para gas natural licuado y $0.2 \%$ para gas licuado de petróleo.

Las correlaciones de Iglesias-Silva y Hall y Spencer y Danner producen desviaciones mayores que el método de estados correspondientes, pero mas bajas que las desviaciones reportadas por Nasrifar y Bolland (2006) y Janish et al. (2007), para predicciones de densidad de líquido saturado con diferentes ecuaciones cúbicas de estado las desviaciones absolutas varían entren $1.16 \%$ y $10.76 \%$. 
Para una comparación de el método de estados correspondientes con la ecuación de estado de Eslami, se seleccionaron tres mezclas binarias de metano y etano que forman el sistema 1 en la Tabla 1 y se aplicó la ecuación de estado analítica de Eslami (2001). Los resultados se representan en la Figura 2. La desviación de la ecuación de Eslami en mayor al 2\%, mientras que el método de estados correspondientes presenta desviaciones menores al $0.1 \%$.

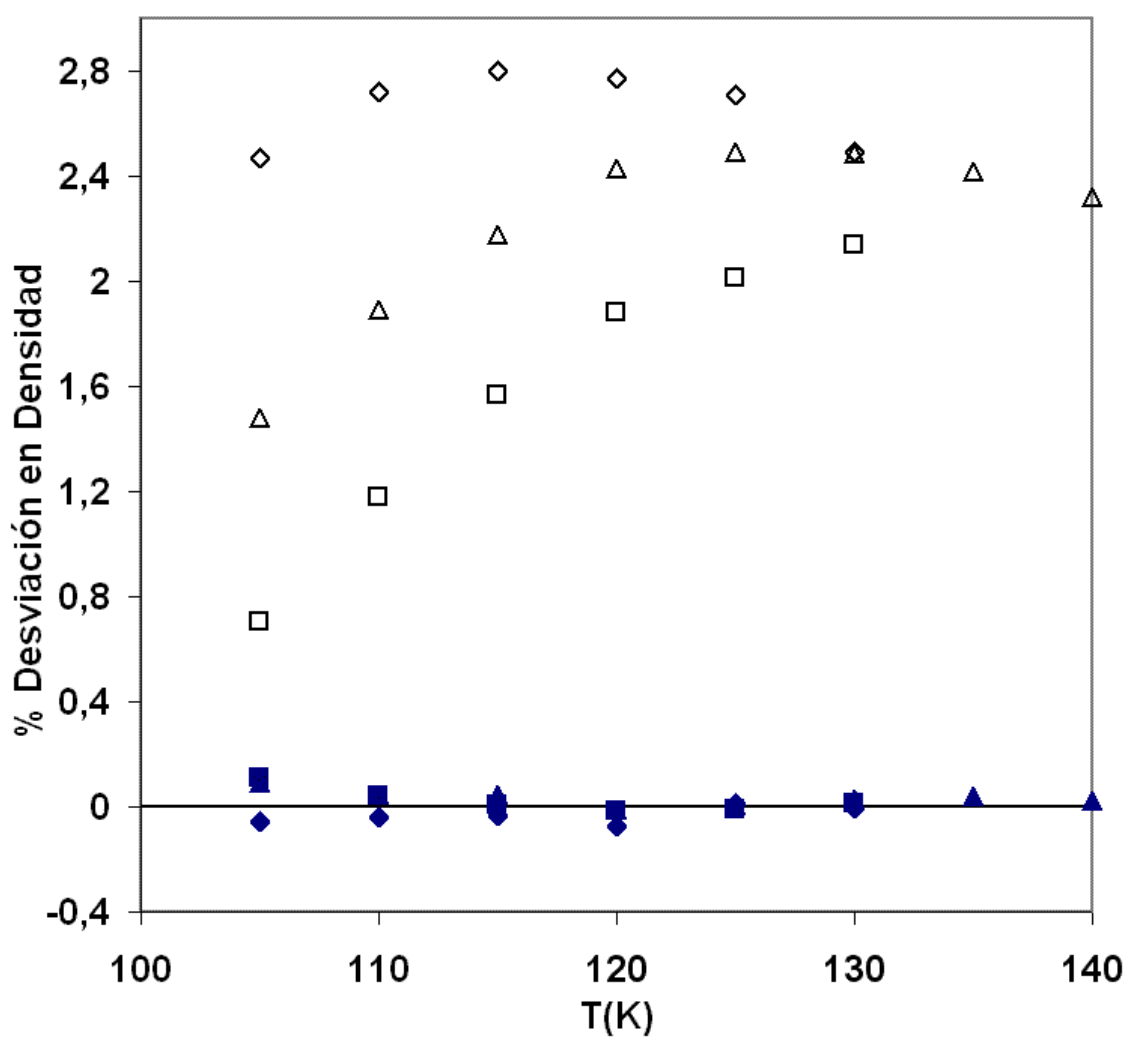

\begin{tabular}{|c|c|c|c|}
\hline \multicolumn{2}{|c|}{ DESV\% } & \multirow{2}{*}{\multicolumn{2}{|c|}{ Eslami, 200 }} \\
\hline TEMP./ & EC & & \\
\hline 105 & 0.1081 & $\square$ & 0.7 \\
\hline 110 & 0.0415 & & 1.175 \\
\hline 115 & 0.0064 & & 1.565 \\
\hline 120 & -0.017 & & 1.88 \\
\hline 125 & -0.012 & & 2.01 \\
\hline 130 & 0.0101 & & 2.14 \\
\hline 105 & $\begin{array}{l}\Delta \quad 0.0926\end{array}$ & $\Delta$ & 1.48 \\
\hline 110 & 0.0473 & & 1.893 \\
\hline 115 & 0.0447 & & 2.18 \\
\hline 120 & -0.013 & & 2.43 \\
\hline 125 & 0.0035 & & 2.49 \\
\hline 130 & 0.0285 & & 2.484 \\
\hline 135 & 0.0409 & & 2.42 \\
\hline 140 & 0.0222 & & 2.32 \\
\hline 105 & -0.059 & $\diamond$ & 2.47 \\
\hline 110 & -0.041 & & 2.72 \\
\hline 115 & -0.032 & & 2.8 \\
\hline 120 & -0.077 & & 2.77 \\
\hline 125 & 0.0135 & & 2.707 \\
\hline 130 & -0.003 & & 2.49 \\
\hline
\end{tabular}

Fig. 2: Desviación de densidad de líquido saturado de mezclas de gas natural. Método de Estados Correspondientes (PEC), AAD\% = 0.03566, BIAS\%=0.1081; Ecuación de Estado de Eslami,

$\mathrm{AAD} \%=2.1562, \mathrm{BIAS} \%=2.8$.

\section{CONCLUSIONES}

El método extendido de estados correspondientes es el método más seguro para la predicción de densidades de gas natural licuado con una desviación menor a $0.1 \%$, y de densidades de gas licuado de petróleo con una desviación menor a $0.2 \%$. Estos resultados muestran que con este método las densidades pueden calcularse dentro del error experimental. A las condiciones de temperatura y presión utilizadas en gasoductos el método extendido de estados correspondientes proporciona las mejores estimaciones de densidad de líquido saturado de gas natural y gas licuado de petróleo. Estos resultados están de acuerdo con los resultados de Mollerup $(1974,1977)$ y McCarthy (1982).

La correlación de Iglesias-Silva y Hall produce desviaciones en densidad menores a $0.46 \%$ y $0.8 \%$ para gas natural licuado y gas licuado de petróleo respectivamente, este resultado es muy bueno para una correlación de dos parámetros y reglas de mezclado de Kay (Nasrifar et al. 1999). Los resultados de la correlación de Spencer y Danner son comparables, pues las desviaciones en densidad son menores a $0.69 \%$ y $0.81 \%$ para gas natural licuado y gas licuado de petróleo respectivamente.

La ecuación de estado de Eslami y las ecuaciones cúbicas predicen la densidad de líquido saturado con distintos grados de precisión, pero siempre mayores entre 1\% y $10 \%$ de desviación, la exactitud aumenta al aumentar la complejidad de la ecuación de estado. Valderrama y Alfaro (2000), revisaron los cálculos de volumen de líquido con ecuaciones cúbicas y concluyeron que para valores precisos de densidad de líquido saturado, las ecuaciones de estado no son la mejor opción. 


\section{AGRADECIMIENTOS}

Al Fondo de Apoyo a la Investigación (FAI) de la Universidad Autónoma de San Luis Potosí por el apoyo recibido que hizo posible la realización de este trabajo.

\section{REFERENCIAS}

Eslami, H.; "Prediction of the Density for Natural Gas and Liquefied Natural Gas Mixtures", AICHE. J.: 47 (11), 2585-2592 (2001)

Estela-Uribe, J.F. y J.P.M. Trusler; "Shape factors for the Light hydrocarbons", Fluid Phase Equilibria: 150-151, 225-234 (1998)

Estela-Uribe, J.F. y J.P.M. Trusler; "Extended Corresponding States Equations of State for Natural Gas Systems", Fluis Phase Equilibria: 183-184, 21-29(2001).

Iglesias-Silva, G.A. y K.R. Hall; "A saturated liquid density equation for refrigerants", Fluid Phase Equilibria: 131, 97-105 (1997).

Janisch, J., G. Raabe y J. Köhler; "Vapor-Liquid Equilibria and Saturated Liquid Densities in Binary Mixtures of Nitrogen, Methane, and Ethane and Their Correlation Using the VTPR and PSRK GCEOS", J. Chem. Eng. Data: 52(5), 1897-1903 (2007).

Klosek, J. y C. McKinley; "Densities of Liquqfied Natural Gas and of Low Molecular Weight Hydrocarbons", Proc. First Int. Conf. On LNG, IGT, 549-560, Chicago (1968)

Leach, J.W., P.S. Chappelear y T.W. Leland; "Use of Molecular Shape Factors in Vapor-Liquid Equilibrium Calculations with the Corresponding States Principle", AIChE J.: 14 (4), 568-576 (1968).

Leland, T.W., J.S. Rowlinson y G.A. Sather; "Statistical Thermodynamics of Mixtures of Molecules of Different Sizes", Trans. Faraday Soc.: 64, 1447-1460 (1968)

Leland, T.W., J.S. Rowlinson y G.A. Sather; "Statistical Thermodynamics of Two-Fluid Models of Mixtures", Trans. Faraday Soc.: 65, 2034-2043 (1969)

McCarthy, R.D.; "Mathematical Models for the Prediction of Liquefied-Natural-Gas Densities", J. Chem. Thermodyn.: 14, 837-854 (1982)

Mollerup, J., y J.S. Rowlinson; "The Prediction of the Densities of Liquefied Natural Gas and of Lower Molecular Weight Hydrocarbons", Chem. Eng. Sci.: 29, 1373-1381 (1974)

Mollerup, J., "Thermodynamic Properties of Natural Gas, Petroleum Gas and Related Mixtures. Part I: Mixed Fluid Densities", Ber. Bunsen-Gesellschaft. Phys. Chem.: 81, 1015-1020 (1977)

Nasrifar, K., y O. Bolland; "Prediction of thermodynamics properties of natural gas mixtures using 10 euations of state including a new cubic two-constant equation of state", J. Pet. Sci. Eng.: 51,253-266 (2006)

Nasrifar, K., S. Ayatollahi y M. Moshfeghian; "An extended saturated liquid density", Fluis Phase Equilibria: 166, 163-181 (1999).

Nasrifar, K., S. Ayatollahi y M. Moshfeghian; "Generalized saturated liquid density prediction method for pure compounds and multicomponent mixtures", Fluis Phase Equilibria: 168, 71-90 (2000).

Nasrifar, K., y M. Moshfeghian; "Vapor-liquid equilibria of LNG and gas condensate mixtures by the Nasrifar-Moshfeghian equation of state", Fluis Phase Equilibria: 200, 203-216 (2002). 
Peng, D.Y. y D.B. Robinson; “A New Two-Constant Equation of State”, Ind. Eng. Chem. Fund.: 15(1), 59-64 (1976)

Rowlinson, J.S. e I.D. Watson; "The Prediction of the Thermodynamic Properties of fluids and fluid Mixtures. I The Prinicple of Corresponding States and its Extensions", Chem. Eng. Sci.: 24, 15651574 (1969).

Rodosevich, J.B y R.C. Miller; "Calculation of LNG Excess Volumes by a Modified Hard-Sphere Model“, Adv. Cryo. Engr.: 19, 339-345 (1974)

Setzmann, U., y W. Wagner; "A new equation of state and tables of thermodynamic properties for methane covering the range from the melting line to $625 \mathrm{~K}$ at pressures up to $1000 \mathrm{MPa}$ ".J. Phys. Chem. Ref. Data: 20, 1061-1155 (1991).

Spencer, C.F. y R.P. Danner; "Improved Equation for Prediction of Saturated liquid Density". J. Chem. Eng. Data: 17 (2), 236-241 (1972).

Soave, G.; "Equilibrium constant from a modified Redlich-Kwong equation of state" Chem. Eng. Sci: 27(6), 1197-1203 (1972).

Valderrama, J.O. y M.A. Alfaro; "Liquid Volumes from Generalized Cubic Equations of State. Take It with Care". Rev. Inst. Fr. Pet. Oil Gas Sci. Technol.: 55(5), 523-531 (2000) 